\title{
T2* dynamic contrast enhanced MR perfusion for cervical cord lesions; does it work?
}

\author{
Mohamed Saied Abdelgawad ${ }^{1 *}$ (D, Mohamed Ihab Samy Reda ${ }^{2}$ and Nermeen Abd El-Monsef Abd El-Maaboud ${ }^{2}$
}

\begin{abstract}
Background: The goal of this work was to assess the value of magnetic resonance (MR) perfusion in narrowing the differential diagnosis of cord lesions.

Thirty eight patients with different cervical cord lesions were involved in this study. This includes 20 males and 18 females, ranging between 13 and 60 years old.

Conventional MR with T2W (axial and sagittal) and pre and post contrast T1W (axial and sagittal) in addition to the T2* MR perfusion sequence were done. The final diagnosis of cervical cord tumors was achieved by biopsy and histopathological diagnosis, while inflammatory lesions were proved by clinical, laboratory data and follow-up for six months.

Results: Neoplastic lesions were found in 13 patients, while 25 patients had inflammatory lesions. Relative cord/cerebral blood volume ( $\mathrm{rCBV}$ ) was significantly higher in neoplastic lesions when compared to non-neoplastic ones $(2 \pm 1.13 \mathrm{vs}$ $1.01 \pm 0.62$ ), respectively. A cutoff value of 1.38 or higher has high sensitivity of $78 \%$ and specificity of $83 \%$ in differentiating between these lesions.
\end{abstract}

Conclusion: $\mathrm{T}^{*}$ is a valuable technique in differentiating neoplastic from non-neoplastic cervical cord lesions.

Keywords: MR perfusion, Cervical cord lesions

\section{Background}

Perfusion imaging with T2* dynamic susceptibility contrast enhancement magnetic resonance imaging (MRI) rely upon the tissue microvasculature. It is performed by injection of MRI-specific contrast material through a venous line and monitoring it as it passes throughout the tissue microvasculature. The tumor grade depends on its tissue vasculature. The tumor vasculature is elevated relative to normal tissues. In high-grade tumors, there is a large number of vessels and a greater volume of blood and rate of perfusion passing through it [1].

In dynamic perfusion MRI with $\mathrm{T} 2 *$ or gradient sequences, the signal level declines in areas with higher contrast concentration due to alterations in local susceptibility. This is different from perfusion with T1-weighted sequence in which the signal strength parallels the high contrast concentration [2].

\footnotetext{
* Correspondence: mselgawad@yahoo.com

${ }^{1}$ Radiology Department, National Liver Institute, Menoufia University, Shebin

El Kom, Egypt

Full list of author information is available at the end of the article
}

New MR systems with strong gradient hardware, allow dynamic examination with at least one image stack repetition per second. These make the visualization of paramagnetic contrast media passing along cord segments possible [3].

Conventional MRI with contrast is basically used for assessment of many different cervical cord lesions; but sometimes, it is very hard to recognize the difference between inflammatory and neoplastic cord lesions; this makes surgical biopsy unavoidable [4].

Neoplastic cord lesions are usually presented as illdefined hyperintense lesions on $\mathrm{T} 2 \mathrm{~W}$ with heterogenous enhancement; astrocytoma composes the majority $[5,6]$.

The cervical cord by far is the commonest site with prediction to eccentric location, while with lesser degree to dorsal segments. Heterogeneous incomplete contrast enhancement is usually seen with obscured margins of the lesion. Intra-lesion cystic changes are sometimes identified [7].

On the other hand, ependymomas are well-defined lesions displaying intense homogeneous enhancement. In 
certain cases, T2W hypointense rim is present around the lesion due to previous hemorrhagic changes, and this is called "cap sign" [8].
Haemangioblastomas characteristically appear as sharply defined T2 hyperintense cystic lesions, harboring intensely enhancing wall nodules [9].
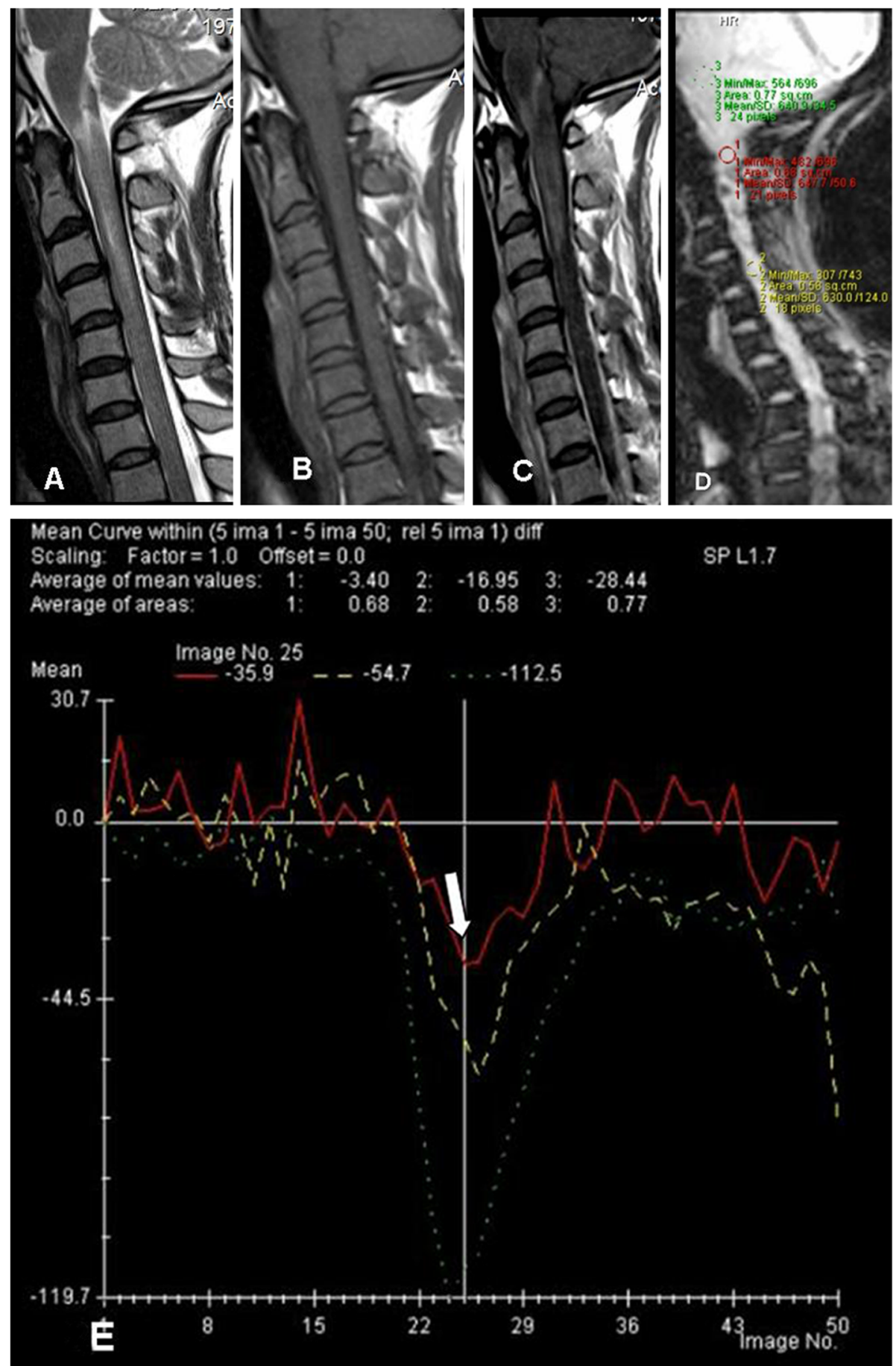

Fig. 1 Thirty-seven-year-old male patient presented by acute onset of bilateral lower limb weakness and neck pain. a Sagittal T2 revealed abnormal T2 hyperintense signal spanning over the cervicomedullary junction till CV4 level. b T1W pre and c post contrast showing faint patchy enhancement. $\mathbf{d}$ rCBV was calculated in relation to the normal lower cervical cord, showing e hypoperfusion (arrow to red curve) compared to the normal cord (yellow curve), suggesting non-neoplastic inflammatory process. Green curve indicates the pons. Initial clinical diagnosis of viral myelitis (zoster) due to appearance of skin eruptions, the patient improved clinically, confirming its non-neoplastic nature 

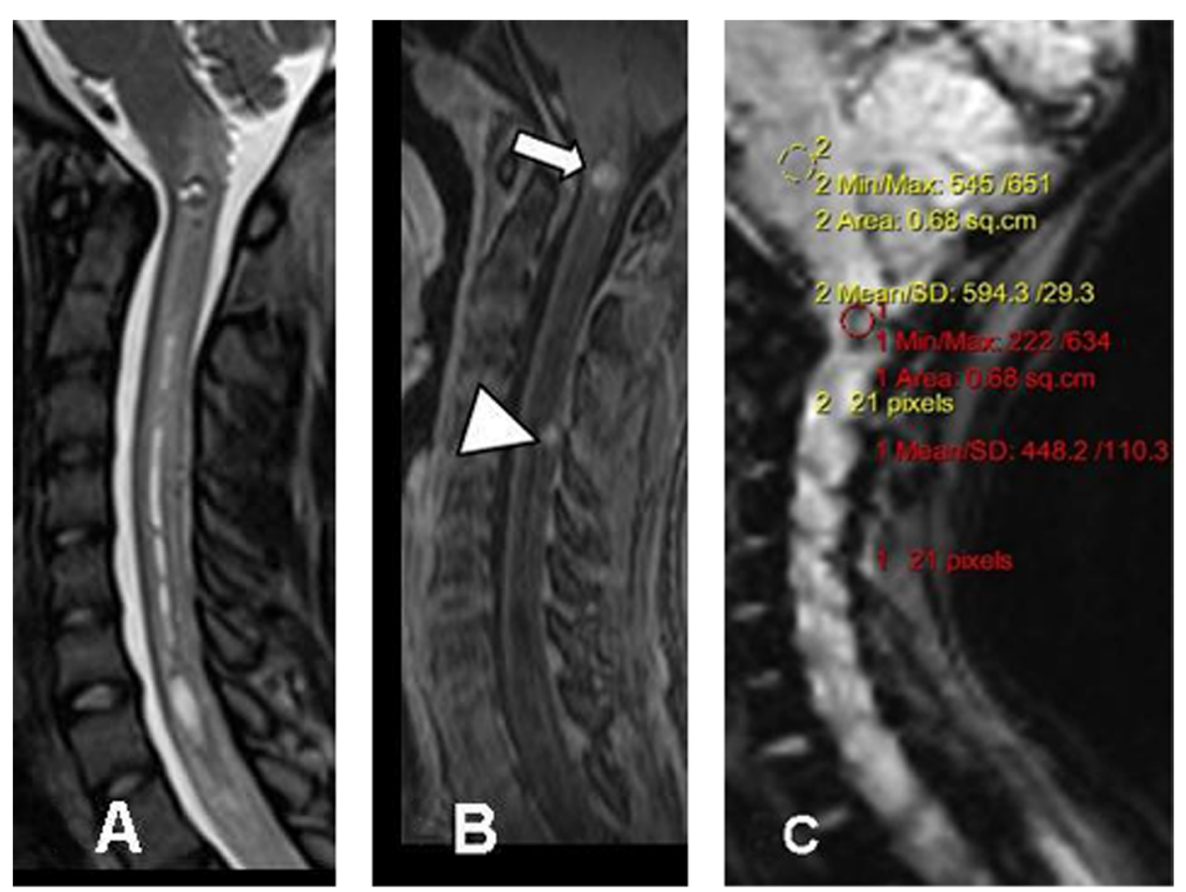

Mean Curve within (34 ima 1 - 34 ima 50; rel 34 ima 1) diff Scaling: Factor $=1.0$ Offset $=0.0$ Average of mean values: $1:-74.51$ 2:-23.43 Average of areas:

1: 0.68

SP R3.8

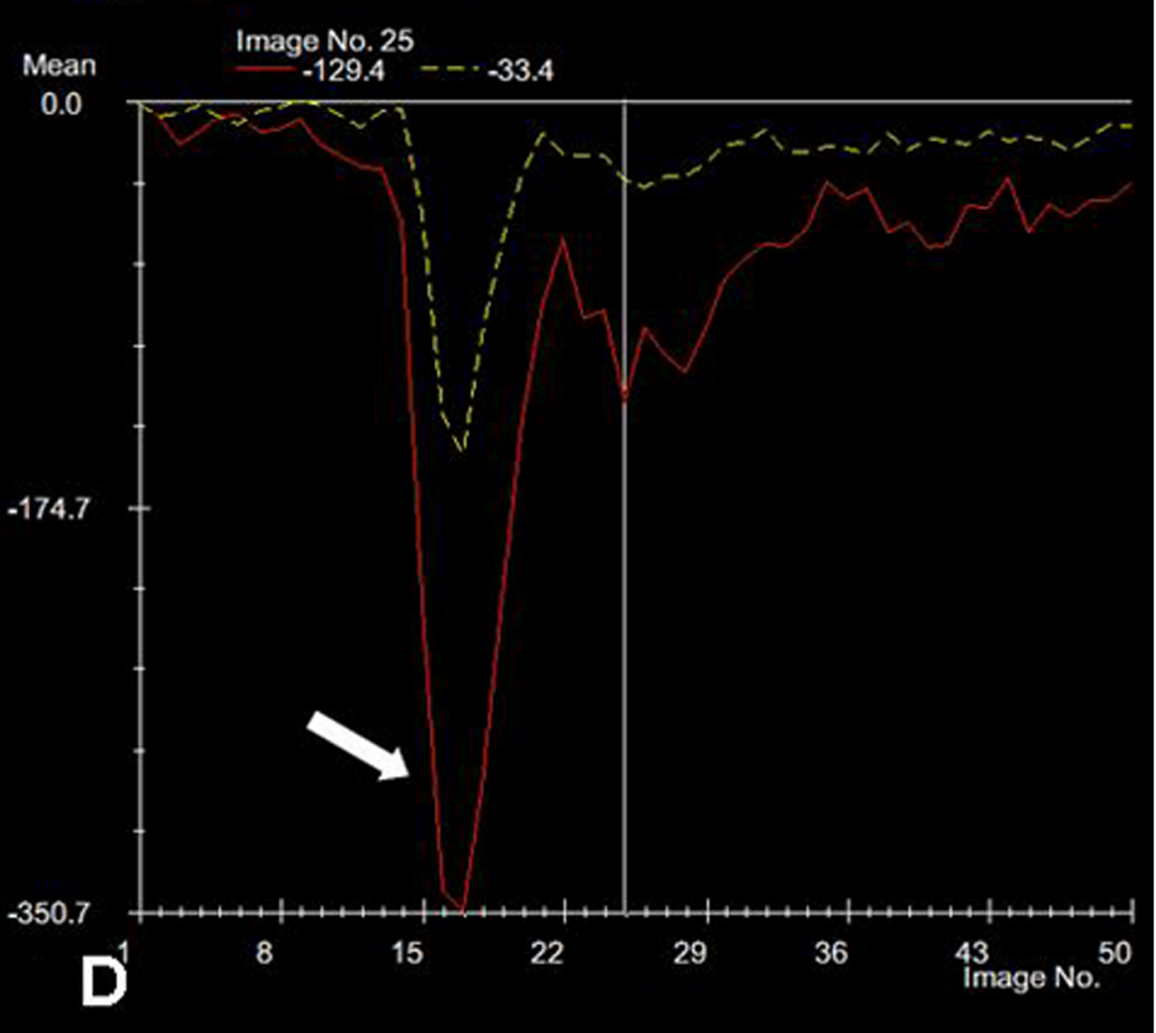

Fig. 2 (See legend on next page.) 
(See figure on previous page.)

Fig. 2 Eighteen-year-old female patient presented by recurrent neck pain and bilateral upper limb numbness with weakness on the right side. a Sagittal T2 and $\mathbf{b}$ T1 post contrast showing small enhancing intramedullary lesion (arrow) located opposite CV2 associated with syrnix extending along the cervical cord and enhancing extra-medullary intra-dural lesion (arrowhead). $\mathbf{c}$ and $\mathbf{d}$ rCBV was calculated in the lesion (arrow to red curve) in relation to the pons (yellow curve), showing a hyperperfusion pattern. The lesion was pathologically diagnosed as anaplastic astrocytoma

Spinal cord deposits are very rare. The top list metastatic neoplasms to the cord are colon, breast, followed by renal neoplasm, and lymphoma. An intense enhancing cord lesion with extensive disproportionate nearby cord edema must be considered in terms of metastatic etiology [10].

Multiple sclerosis commonly affects the cervical cord, with preferential involvement of the posterior and lateral cord aspects [11].

Cord transverse myelitis usually affects its inner portion, extending for more than three adjacent vertebrae length [12].

Neuromyelitis optica (NMO) by the same way presented by long-segment cervical cord hyperintense lesion reaching up to three contiguous vertebrae, but usually there is optic nerve affection [13].

Post irradiation myelitis often appears as a large area of T2W hyperinensity at the irradiated cord segment and shows disproportionate irregular post contrast enhancement [14].

The aim was to evaluate the performance of $\mathrm{T} 2 * \mathrm{dy}$ namic susceptibility contrast-enhanced MRI perfusion in different cervical cord lesions.

\section{Methods}

\section{Patients}

The patients' consents were regulated by the principles of research board, confirming medical records privacy of patients. Thirty eight patients with different lesions affecting the cervical cord were involved in the study. This included 20 males and 18 females, ranging between 13 and 60 years old (mean age $=38$ years, Figs. 1, 2, 3 and 4 ).

Patients with proved diagnosis of either neoplastic cord masses or non-neoplastic/inflammatory cord lesions were included in this study, while, traumatic cord cases or vascular malformations were excluded.

The final diagnosis of cervical cord tumors was proved by biopsy and histopathological diagnosis (13 patients); while inflammatory lesions ( 25 patients) were confirmed by clinical, laboratory data, and follow-up for six months.

A detailed clinical data was taken from all patients, consisting of personal data, past history, and neurological complaints.

The examination for all patients were done by a closed MRI unit 1.5 T (Avanto, Siemens, Erlangen, Germany), without complications.

\section{Conventional MRI}

In the conventional MRI, (1) T2 TSE (axial and sagittal) with TR of $3500 \mathrm{~ms}$, TE of $88 \mathrm{~ms}$, and acquisition time of $2 \mathrm{~min} 35 \mathrm{~s}$ was done for each sequence; (2) pre and post contrast T1 SE (axial and sagittal) with TR of 640 $\mathrm{ms}$, TE of $10 \mathrm{~ms}$ with acquisition time of $3 \mathrm{~min} 23 \mathrm{~s}$ was done for each sequence. A slice thickness of $1 \mathrm{~mm}$, a gap of $0.3 \mathrm{~mm}, 256 \times 256$ acquisition matrix, and $\mathrm{FOV}=250 \mathrm{~mm}$ was used.

\section{Perfusion images}

The dynamic $\mathrm{T} 2 *$ perfusion was acquired with a single shot, gradient-echo, echo-planar imaging sequence. TR/ $\mathrm{TE}=1520 / 32 \mathrm{~ms}$; flip angle, $80^{\circ} ; \mathrm{FOV}=230 \mathrm{~mm}$; matrix = $96 \times 128$; single mid-sagittal slice with a thickness of 10 $\mathrm{mm}$; number of scans 50 , during the first pass of contrast "Dotarem". The volume of contrast was calculated as 0.1 $\mathrm{mmol} / \mathrm{Kg}$ for a maximum of $20 \mathrm{ml}$. The total dose was at flow velocity of $5 \mathrm{ml} / \mathrm{s}$, then a bolus injection of saline (20 $\mathrm{ml}$ ), with an automatic injector through an 18- to 20-gauge needle. The scan time for the sequence was $1 \mathrm{~min} 44 \mathrm{~s}$.

Post processing assessment done by a dedicated software package (syngo neuro perfusion evaluation). Perfusion mean curves were created at different regions of interests (ROIs) including the areas of interest and normal-appearing cervical cord or pons. Then comparative curves were made (Fig. 5).

\section{Results}

The results are summarized in Table 1.

\section{Neoplastic lesions}

Perfusion examinations were achieved in 13 cases with intramedullary neoplasms. We measured tumor relative blood volume (rCBV) in comparison to the sound cord in 9 cases. We computed the rCBV between the cord lesions and the pons in four cases where the tumors are extending along the whole cervical cord. The cases compared to the normal cord showed increased rCBV except one lesion. The estimated rCBV ratio was from 1.08 to 4.5 , with highest $\mathrm{rCBV}$ found in the case of glioblastoma multiforme (GBM). Only one neoplastic lesion was hypoperfused $(\mathrm{rCBV}=0.54)$; this lesion showed minimal progression in size when reviewed from a study done 10 years ago, suggesting low grade nature of the lesion.

We used the normal-appearing cord either above or below the cervical cord lesions, or the normal pons of 

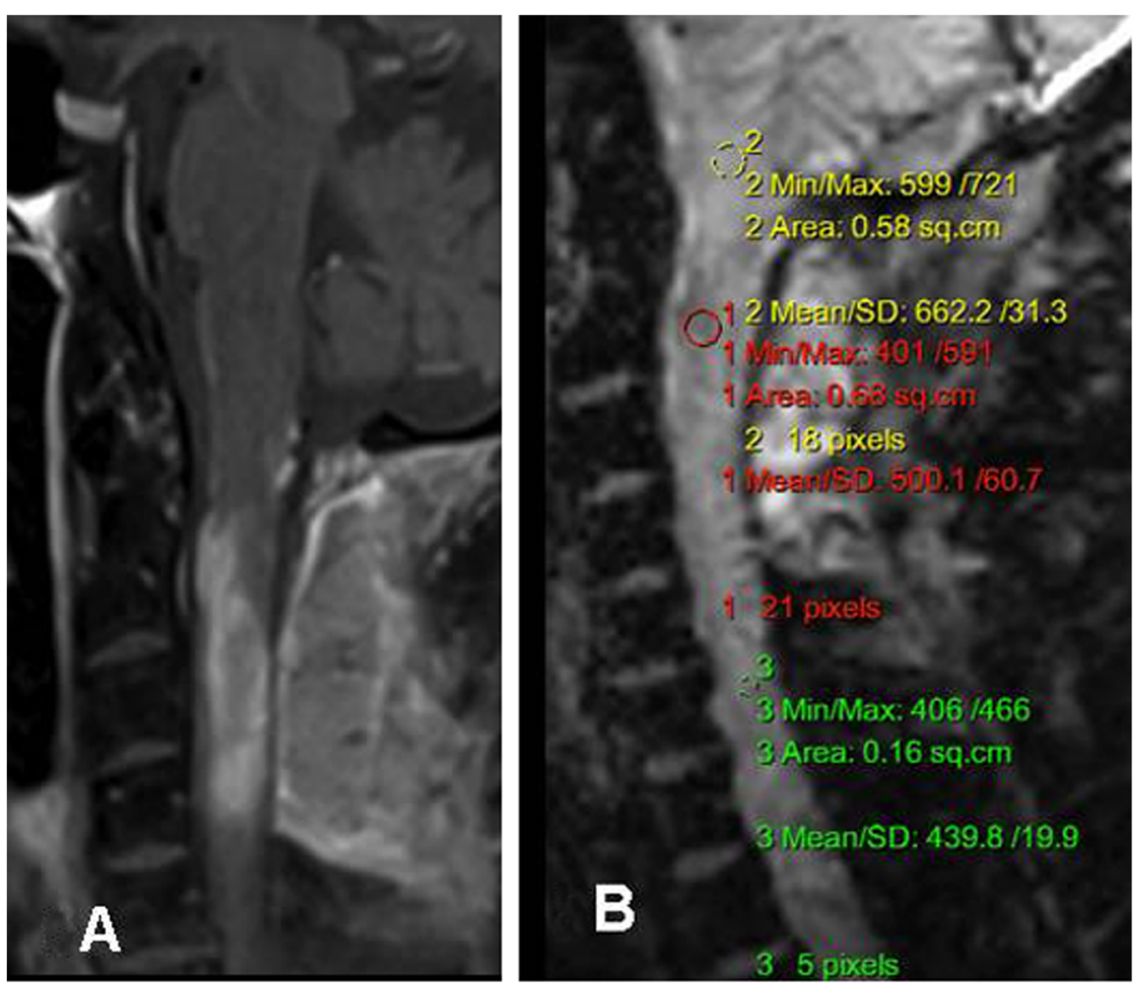

Mean Curve within (22 ima 1.22 ima 50; rel 22 ima 1) diff

Scaling: Factor $=1.0$ Offset $=0.0$

Average of mean values: $1:-48.60$

2:-31.57

3:-25.31

SP L3.5

Average of areas:

1: 0.68

2. 0.58

3. 0.16

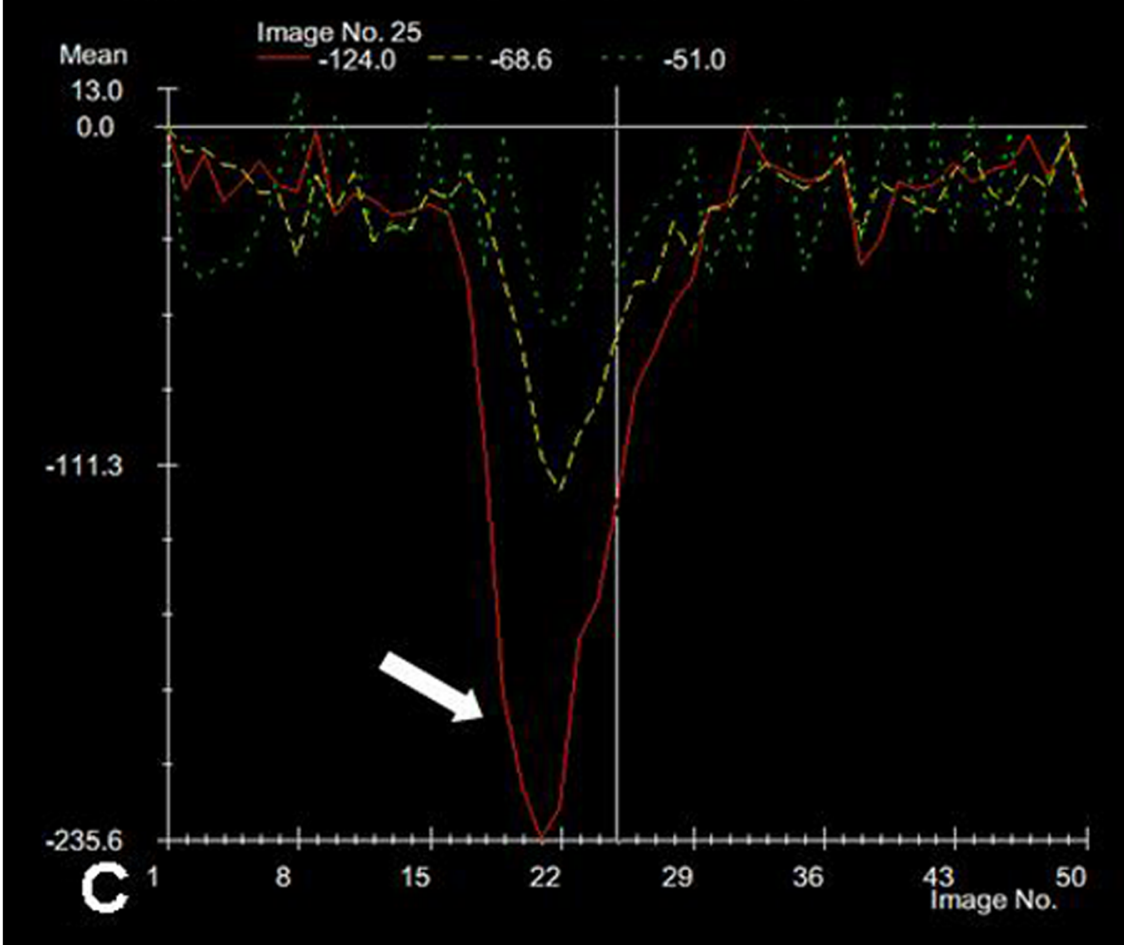

Fig. 3 (See legend on next page.) 
(See figure on previous page.)

Fig. 3 Sixty-year-old male patient presented by recurrent neck pain, bilateral upper limb numbness, and weakness a year after surgical resection of astrocytoma and post operative radiotherapy. a Sagittal T1 post contrast showing irregular enhancing intramedullary lesion at CV2 till CV4 at the surgical bed. $\mathbf{b}$ and $\mathbf{c}$ rCBV shows hyperperfusion of the lesion (arrow to red curve) relative to the normal lower cervical cord (green curve). Proved by biopsy to recurrent anaplasic astrocytoma

the same patients in this study as an internal reference "control".

We found that the normal range of the healthy cord/pons ratio rCBV calculated in 30 cases was about $0.39 \pm 0.12$.

When comparing the $\mathrm{rCBV}$ in the 4 neoplastic lesions which were involving the whole cervical cord and the pons $\mathrm{rCBV}$, the ratio was ranging from 0.7 to 2.5 , suggesting a hyperperfusion pattern.

Then we recalculated rCBV in tumors compared to those of the pons in the 13 cases which were pathologically proved. After that, we compared signal drop ratio between low and higher grade lesions. We observed more signal intensity drop in high-grade tumors than low-grade ones. But we found it statistically insignificant (Table 2).

\section{Non-neoplastic lesions}

Perfusion was done in 25 non-neoplastic lesions. Twenty two cases had focal "non-diffuse" lesion; so we compared rCBV to the normal cord. Eighteen of these 22 patients were either isoperfused or hypoperfused. Only four cases with myelitis were hyperperfused ( $\mathrm{rCBV}=1.5,1.75,1.8$, and 2).

Three cases had diffuse abnormal signals, so we measured the rCBV as compared to the pons. The ratio of these lesions to the pons was $0.35,0.5$, and 0.58 in a case with NMO; the second was transverse myelitis and a third case of post irradiation changes, respectively.

\section{Neoplastic versus non-neoplastic lesions}

Significant high rCBV ratio was found in neoplastic masses targeting the cord than those of non-neoplastic etiology, which can help in differentiating between both categories (Table 3).

The suggested cutoff value (1.38 for rCBV) resulted in a sensitivity of $78 \%$ and a specificity of $83 \%$. Area under curve for rCBV was high (0.845). That was statistically significant ( $p$ value 0.003 .

\section{Discussion}

After extended review of the literatures, publications regarding cord by MR in vivo in humans are scarce, principally on account of the technical difficulties.

The difficulties for MR perfusion in the cervical/cervicomedullary region include the intrinsic susceptibility artifacts as well as motion swallowing artifacts, CSF pulsation, and partial volume impacts [15].
In our study, a significant high perfusion values (rCBV) of the intramedullary tumors than in non-neoplastic lesions was found. The rCBV mean for neoplasms was 2 when compared to the normal cervical cord above or below the lesion, this value is fundamentally higher than the non-neoplastic category where their mean value of rCBV was 1 ( $p=0.003)$.

This is similar to an earlier research of brain lesions that revealed increased rCBV ratio in cerebral gliomas than in demyelinating lesions [16] and matching with the observation listed by Xiang et al. [15] using a perfusion imaging to differentiate intramedullary cervical neoplasm from tumor-like masses [15].

In a publication by Liu et al. [17], for glioblastoma diagnosed by autopsy from upper cervical spinal cord, they observed an increase in $\mathrm{rCBV}$ which suggested a highgrade glioma.

Absence of frank angiogenesis and hypoperfusion sequel to diminished metabolism in the nonneoplastic lesions, explained the difference in tissue perfusion in malignant lesions and inflammatory processes [15].

We noticed that three of our cases with myelitis were slightly hyperperfused; this was similar to an observation noted in a study done by R Hourani et al. [18] who evaluated the value of perfusion MR in characterizing neoplasms from other brain issues. He reported a mean rCBV value in two cases with demyelinating lesions to be $1.3+0.49$. This could be explained by the transient hyperemia occurring in the acute phase of myelitis. A cutoff value equal to or higher than 1.38 of rCBV compared to the normal cord had $78 \%$ sensitivity and $83 \%$ specificity to distinguish neoplastic from the nonneoplastic process.

Calculation of the rCBV ratio is the hardest point, which is an important imaging index in brain perfusion studies. In the brain, the rCBV ratio is calculated by dividing CBV values of a lesion by the CBV value of normal-appearing brain at the other side.

This prerequisite, however, may be non-applicable in diffuse infiltrating lesions that can affect most of the cord/medulla with subsequent erroneous ratio measurements [15].

There are controversies in the acquisition plane selection. Traditionally, axial orientation is frequently used for brain perfusion acquisitions, and ROI is placed in the parenchyma of the unaffected cerebral hemisphere for rCBV and ratio calculations. 

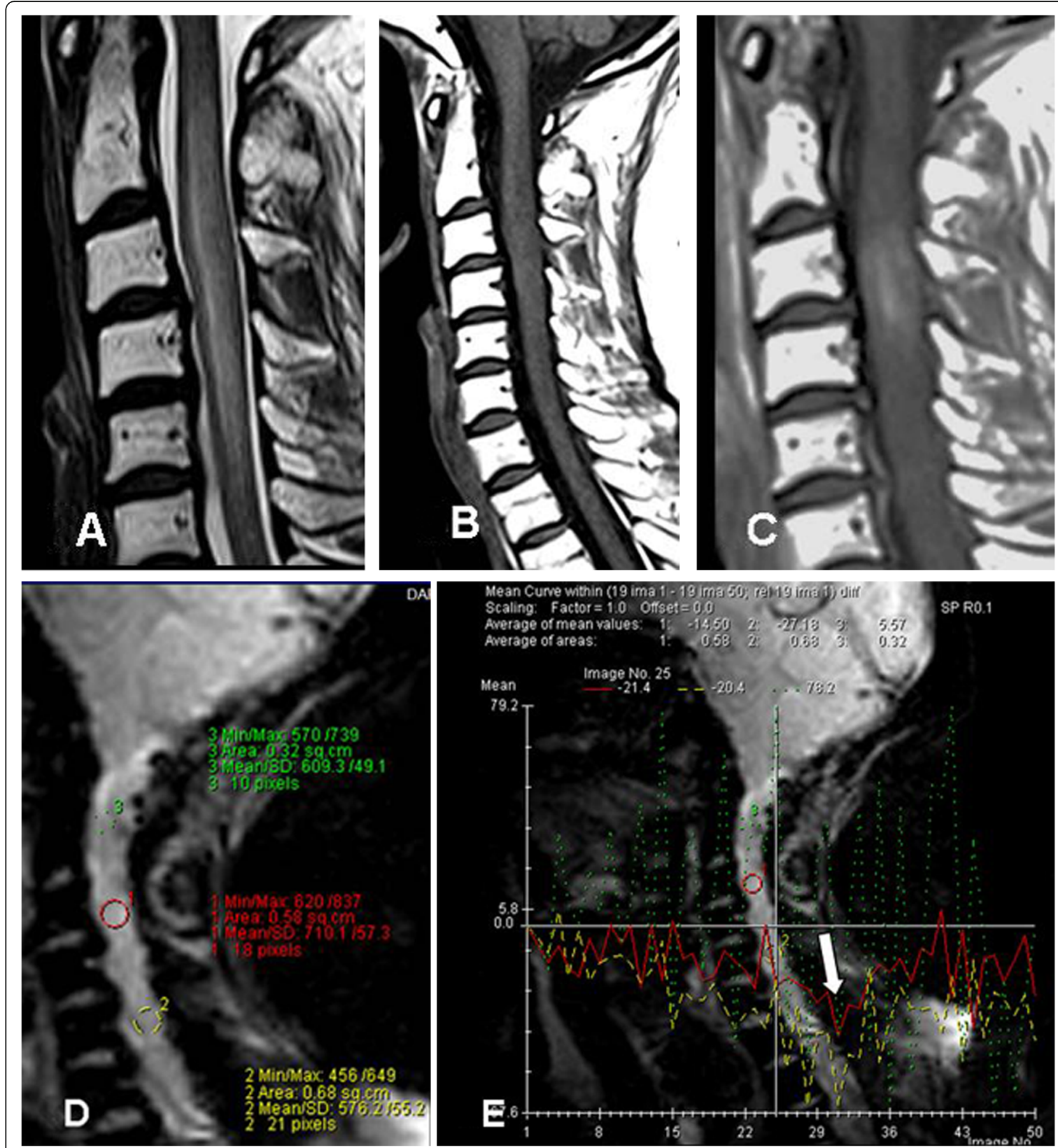

Fig. 4 Fifty-two-year-old female patient presented by neck pain and bilateral upper limb numbness, 3 months after stopping neck irradiation for oral SCC. a Sagittal T2 abnormal hyperintense signal extending from CV2 down to CV6. b Sagittal T1 pre and c post contrast showed mildly enhancing mass like lesion. $\mathbf{d}$ and e rCBV showing hypoperfusion of the lesion (arrow to red curve) in comparison to the normal cervical cord (green and yellow curves). Post irradiation changes were suggested. The lesion regressed on follow up confirming the diagnosis.

However, cervical intramedullary masses may affect multiple segments and also involve the medulla/cord on both sides. Thus, the axial scanning will not assess the entire lesion, with the reference ROI being placed in an adjacent, perilesion part that will not exclude an underestimation of pathologic changes [19]. 

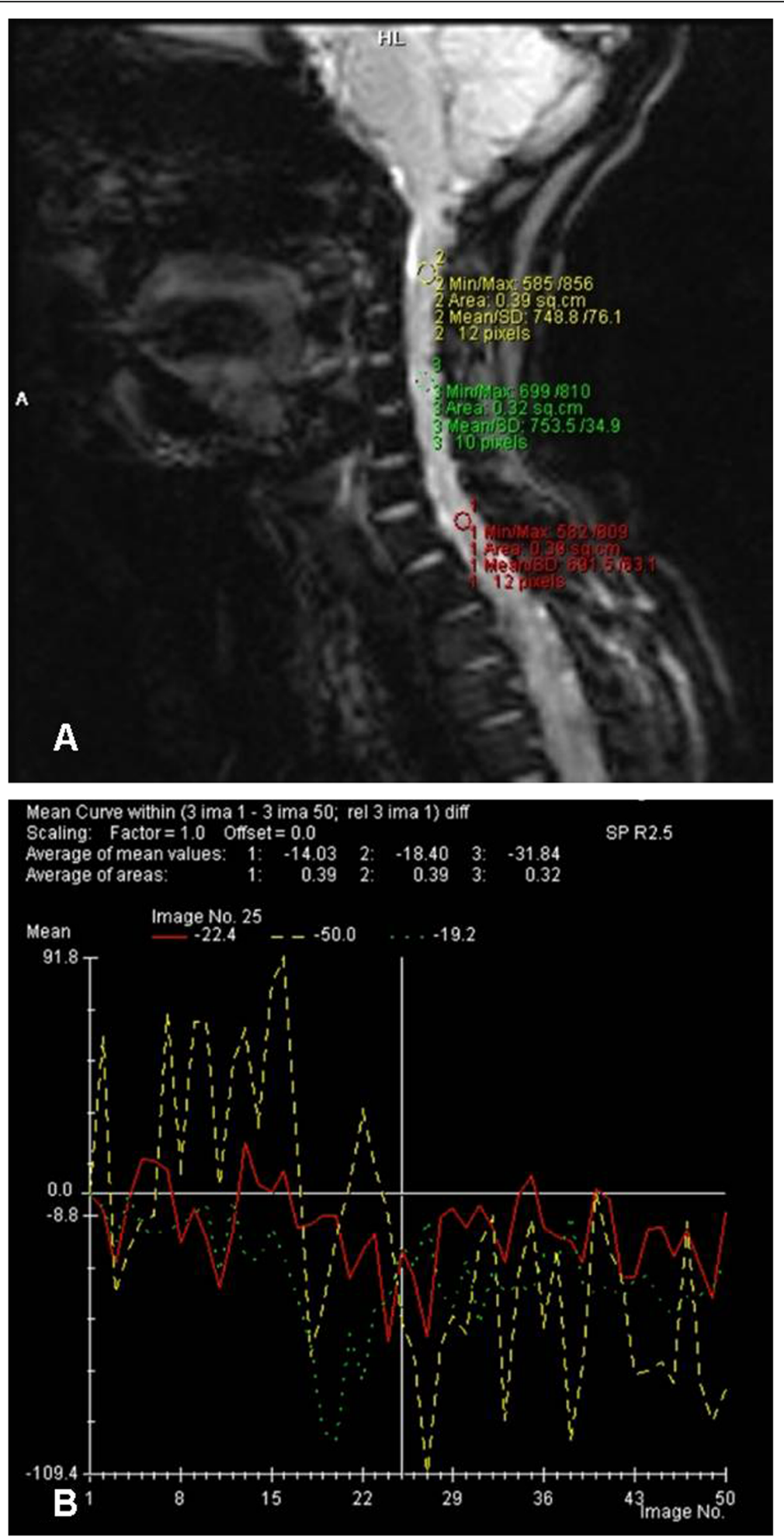

Fig. 5 Demonstration of how post processing assessment method was done by dedicated software. Perfusion mean curves were created by placement of the ROI on sagittal perfusion source (a) to create time intensity curves (b). 
Table 1 Distribution of the studied cases according to final diagnosis and histopathology $(n=38)$

\begin{tabular}{lll}
\hline Pathology & No. of patients $(n=38)$ & Percentage (\%) \\
\hline Non-neoplastic & 25 & 65.79 \\
Myelitis & 12 & 31.57 \\
MS & 9 & 23.68 \\
Post irradiation & 4 & 10.54 \\
Neoplastic & 13 & 34.21 \\
Ependymoma & 5 & 13.16 \\
Fibrillary astrocytoma & 2 & 5.26 \\
Anaplastic astrocytoma & 3 & 7.89 \\
GBM & 2 & 5.26 \\
Metastasis & 1 & 2.64 \\
\hline
\end{tabular}

We selected the sagittal acquisition in our cases to bypass this problem. We assessed the rCBV relative to the pons in four cases with tumors, where the lesions were involving the whole cervical cord. In our study, the average range of normal cervical cord/pons ratio calculated in 30 cases was about 0.3. After measuring the $\mathrm{rCBV}$ in these four cases, the resulting ratio was ranging from 0.7 to 2.5 , suggesting hyperperfusion of these lesions. The rCBV to the pons was calculated in three cases with tumor-like demyelinating lesions. The ratios were $0.35,0.5$, and 0.583 in a case with NMO, a case with transverse myelitis, and a third case of post irradiation changes respectively.

This was matching with the recommendations of Liu et al. [17]; they suggested that the sagittal acquisition may be a helpful approach for perfusion acquisition in cervical cord assessment as it can evaluate the entire lesion and the likelihood of utilizing the cerebellum for the placement of the reference ROI [15].

\section{Conclusion}

Dynamic MR perfusion is a new hopeful method in assessment of lesions within cervical cord that can help in the differentiation between cervical neoplasm and the

Table 2 Mean values (mean \pm SD) and statistical results of regional cord blood volume in tumors and the pons.

\begin{tabular}{llll}
\hline $\begin{array}{l}\text { Ratio between perfusion in tumors } \\
\text { and perfusion of the pons }\end{array}$ & Min.-Max. & Mean \pm SD & Median \\
\hline $\begin{array}{l}\text { In low-grade tumors } \\
(n=5)\end{array}$ & $0.38-2$ & $0.98 \pm 0.62$ & 0.86 \\
$\begin{array}{l}\text { In high-grade tumors } \\
(n=8)\end{array}$ & $0.41-2.5$ & $1.32 \pm 0.8$ & 1 \\
\hline $\begin{array}{l}Z \text { value for Mann Whitney test, } 0.456 ; p \text { value, } 0.322 \\
\text { at } p \leq 0.05\end{array}$
\end{tabular}

Table 3 Comparison between neoplastic and non-neoplastic groups according to rCBV compared to normal cord

\begin{tabular}{lllll}
\hline & $\begin{array}{l}\text { Non-neoplastic } \\
(n=22)\end{array}$ & $\begin{array}{l}\text { Neoplastic } \\
(n=9)\end{array}$ & $Z$ & $p$ \\
\hline rCBV & & & $3.022^{*}$ & $0.003^{*}$ \\
Min.-Max. & $0.17-2$ & $0.54-4.5$ & & \\
Mean \pm SD & $1.01 \pm 0.62$ & $2 \pm 1.13$ & & \\
Median & 1.0 & 2 & & \\
\hline
\end{tabular}

$Z Z$ value for Mann Whitney test

* Statistically significant at $p \leq 0.05$

tumor-like lesions. Although there was a difference in signal drop during dynamic perfusion between highgrade neoplasms and low-grade ones, it was statistically insignificant.

\section{Abbreviations}

DSC-MRI: Dynamic susceptibility contrast-magnetic resonance imaging; IATM: Idiopathic transverse myelitis; NMO: Neuromyelitis optica; PI: Perfusion imaging; rCBV: Relative cord \cerebral blood volume

\section{Acknowledgments \\ Not Applicable}

\section{Authors' contributions}

ME carried out cases on workstation and selection of research cases, prepared the figures for case demonstration, and was involved in the writing and review of the research. MI put the idea and took part in the selection of cases, figure preparation, and follow-up of cases. NA took part in the selection of cases and wiring the cases. All authors read and approved the final manuscript.

\section{Funding}

This study had no funding from any resource.

\section{Availability of data and materials}

The datasets used and/or analyzed during the current study are available from the corresponding author on reasonable request.

\section{Ethics approval and consent to participate}

All procedures followed were in accordance with the ethical standards of the responsible committee on human experimentation, the Institutional Review Board (IRB) of Alexandria General Hospital on 14th February 2017 and with the Helsinki Declaration of 1964 and later versions. The committee's reference number is unavailable (not applicable). No consent was obtained from the patients since it was a retrospective study.

\section{Consent for publication}

All patients included in this research gave written informed consent to publish the data contained within this study.

\section{Competing interests}

The authors declare that they have no competing interests.

\section{Author details}

${ }^{1}$ Radiology Department, National Liver Institute, Menoufia University, Shebin El Kom, Egypt. ${ }^{2}$ Radiology Department, Alexandria Faculty of Medicine, Alexandria University, Alexandria, Egypt.

Received: 26 November 2019 Accepted: 28 November 2019

Published online: 16 December 2019

References

1. Brandão LA, Shiroishi MS, Law M (2013) Multimodality approach with diffusion-weighted imaging, diffusion tensor imaging, magnetic resonance spectroscopy, dynamic susceptibility contrast and dynamic contrast enhanced magnetic resonance imaging. Mag Reson Imaging Clin N Am 21: 199-239 
2. Essig MSM, Nguyen TB (2013) Perfusion MRl: the five mostly asked questions. AJR 200:919-930

3. Paulson ESSK (2008) Comparison of susceptibility weighted contrast enhanced MR methods: recommendations of measuring relative cerebral blood volume in brain tumors. Radiology 249:601-613

4. Fanous Andrew A, Olszewski Nathan P et al (2016) Idiopathic transverse myelitis mimicking an intramedullary spinal cord tumor. Case Reports Pathol:1-8

5. Sun B, Wang C, Wang J, Liu A (2003) MRI features of intramedullary spinal cord ependymomas. J Neuroimaging 13:346-351

6. Baleriaux DL (1999) Spinal cord tumors. Eur Radiol 9:1252-1258

7. Lowe GM (2000) Magnetic resonance imaging of intramedullary spinal cord tumors. J Neurooncol 47:195-210

8. Miyazawa N, Hida K, Iwasaki Y, Koyanagi I, Abe H (2000) MRI at 1.5 T of intramedullary ependymoma and classification of pattern of contrast enhancement. Neuroradiology 42:828-832

9. Baker KB, Moran CJ, Wippold FJ 2nd, Smirniotopoulos JG, Rodriguez FJ, Meyers SP et al (2000) MR imaging of spinal hemangioblastoma. AJR Am J Roentgenol 174:377-382

10. Reddy P, Sathyanarayana S, Acharya R, Nanda A (2003) Intramedullary spinal cord metastases: case report and review of literature. J La State Med Soc 155:44-45

11. Tench CR, Morgan PS, Jaspan T, Auer DP, Constantinescu CS (2005) Spinal cord imaging in multiple sclerosis. J Neuroimaging 15:94S-102S

12. Choi KH, Lee KS, Chung SO et al (1996) Idiopathic transverse myelitis: MR characteristics. AJNR 17:1151-1160

13. Filippi M, Rocca MA (2004) MR imaging of Devic's neuromyelitis optica. Neurol Sci 25:S371-S373

14. Michikawa M, Wada Y, Sano M, Uchihara T, Furukawa T, Shibuya H et al (1991) Radiation myelopathy: significance of gadolinium-DTPA enhancement in the diagnosis. Neuroradiology 33:286-289

15. Liu X, Tian W, Kolar B, Hu R, Huang Y, Huang J et al (2014) Advanced MR diffusion tensor imaging and perfusion weighted imaging of intramedullary tumors and tumor like lesions in the cervicomedullary junction region and the cervical spinal cord. J neurooncol 116:559-566

16. Cha S, Pierce S, Knopp EA, Johnson G, Yang C, Ton A et al (2001) Dynamic contrast-enhanced T2*-weighted MR imaging of tumefactive demyelinating lesions. Am J Neuroradiol 22:1109-1116

17. Liu X, Germin Bl, Ekholm S (2011) A case of cervical spinal cord glioblastoma diagnosed with MR diffusion tensor and perfusion imaging. J Neuroimaging 21:292-296

18. Hourani R, Brant L, Rizk T, Weingart JD, Barker PB, Horská A (2008) Can proton MR spectroscopic and perfusion imaging differentiate between neoplastic and non-neoplastic brain lesions in adults? AJNR Am J Neuroradiol 29(2):366-372

19. Alexander AL, Lee JE, Wu YC, Field AS (2006) Comparison of diffusion tensor imaging measurements at 3.0 T versus $1.5 \mathrm{~T}$ with and without parallel imaging. Neuroimaging Clin N Am 16:299-309

\section{Publisher's Note}

Springer Nature remains neutral with regard to jurisdictional claims in published maps and institutional affiliations.

\section{Submit your manuscript to a SpringerOpen ${ }^{\circ}$ journal and benefit from:}

- Convenient online submission

- Rigorous peer review

- Open access: articles freely available online

- High visibility within the field

- Retaining the copyright to your article

Submit your next manuscript at $\boldsymbol{\nabla}$ springeropen.com 